\title{
Articulatory postures and forward models in American Sign Language: Linguistic and neuroscience evidence
}

\author{
David P. Corina (Department of Linguistics, \\ Center for Mind and Brain, University of California, Davis) \\ dpcorina@ucdavis.edu
}

\begin{abstract}
This paper proposes a forward model of sign language processing in which abstract holistic postural forms guide the production and recognition of signed language. Internal linguistic evidence based on an ASL corpus and morphological compounds is offered to support the utility of this construct. External evidence from child language acquisition, psycholinguistic and neurolinguistic studies are provided to highlight the importance of postural forms in the production and recognition of American Sign Language. I argue that abstract articulatory postural forms play a role in forward modeling accounts of sign language production and recognition.
\end{abstract}

Keywords: sign language production, sign language perception, articulatory postures, psycho- and neurolinguistics, forward models

\section{Introduction}

A casual observer watching a user of a signed language is struck by the seemingly constant movement of the hands, arms and face. Indeed, the entire body serves as the articulator in the fluent expression of signs. Sign linguists too have been captivated by the movements observed in sign languages with some treating this property as a distinct segment class (see for example, Liddell and Johnson 1986; Sandler and Lillo-Martin 2006). Explication of the differences in types of movements (e.g., path movement, internal movement and transitional movements) have been important in our current understanding of sign language structure. However, it is curious that given the centrality of movement in signed languages, the inventory of such movements is surprisingly limited. ${ }^{1}$ For example, linguistic theories that posit path-movements propose essentially four distinct movements (see Table 1$)^{2}$

While movement inventories are further enriched by dynamic changes in the shape of the hands and fingers, given the potential capacity for creating perceptually distinct movements, it may be surprising to learn that sign languages generally have restricted sets of movements; arm movements $(\mathrm{n} \approx 4)$, hand movements $(\mathrm{n} \approx 3)$ and finger movements $(\mathrm{n} \approx 3)$. Moreover, not only are the inventories of movement types observed in signed languages quite limited,

1. Here I am referring to core lexical signs and exclude morphologically complex signs and classifier constructions.

2. American Sign Language includes a "7" movement.

Corina, David P. (2021). Articulatory postures and forward models in American Sign Language: Linguistic and neuroscience evidence. FEAST 4: 61-73. https://doi.org/10.31009/FEAST.i4.05 
Table 1: Sign Language Path Movements.

1 A straight movement of the hand to the signer's body.

2 A straight movement of the hand away from the signer's body.

3 An arced movement of the hand.

4 Circling movements of the hand(s).

the combinatorial use of movements in lexical signs is highly constrained. Syllabic and morphemic constraints on word formation in signed languages conspire to limit the articulatory content within a sign (Battison 1978; Stokoe 1960; Sandler and Lillo-Martin 2006). For example, the generalization that there is one major body location per morpheme limits the extent of potential movements. As the majority of signs in sign languages like ASL tend to be monosyllabic and monomorphemic, the movements tend to be quite simple, restricted in form and in displacement. Moreover, devices which might be used to modify these restricted movements, such as repetition, appear underutilized. As Channon (2002) notes, while rhythmic repetition is commonly observed in signed languages, the consequences of these repetitions is seldom lexically contrastive. While non-repetition can contrast with simple repetition (e.g., ON/WARN, ALMOST/EASY etc.), two, three, four or more repetitions of an action in a sign does not change its lexical meaning.

So, we are faced with a conundrum, one the one hand, movement appears to be central to expression of signed languages, yet the actual inventory of movements is really quite modest, the combination of movements is highly constrained, and basic processes like the repetition of a movement, which in principle could be generatively used in the formation of contrastive sign forms, is surprisingly underutilized.

\section{Signs as postures}

Here I will offer an alternative conception of basic lexical forms used in sign languages, one that argues that we should treat many sign forms as postures and that the act of signing entails achieving a specific postural form. I want to be clear; I am not suggesting that movements play no role in linguistic and perceptual processes underlying sign language formation and recognition. Rather, I suggest that the current focus on movement has overshadowed additional central properties of sign languages. This line of reasoning, forces us to consider the importance of moments of stasis in sign languages and holistic postural forms that are enacted at these moments in time. We begin with a definition of a canonical posture. See also Figures 1 and 2 for examples of signs.

(1) The body configuration that is achieved when the signers' hand(s) are in physical contact with another part of the body. The entire bodily form that is created by this juxtaposition of arms and body constitutes a posture.

\subsection{Contacted locations}

In the postural account of signed language, the presence of a "contacted location" in a canonical posture holds a special status. Below I provide both internal and external linguistic ev- 
Figure 1: Signs that exhibit canonical postures: FATHER (left), CORRECT (right)

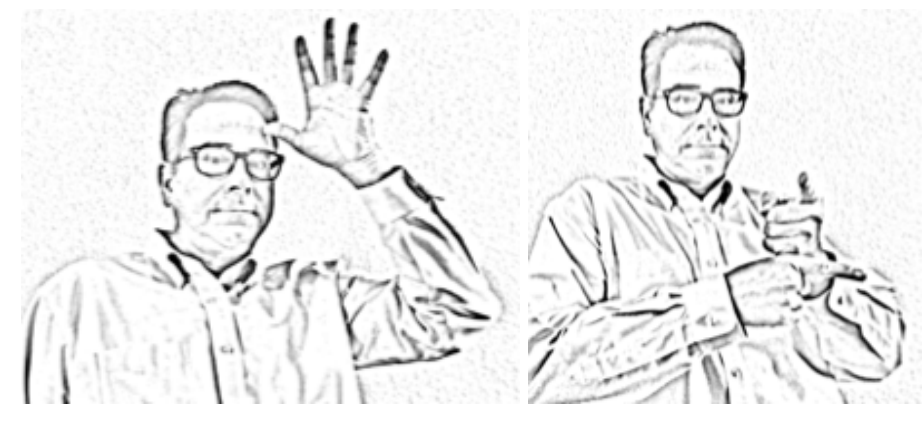

Figure 2: Signs that lack a canonical posture: REACH-TO (left), BICYCLE (right)

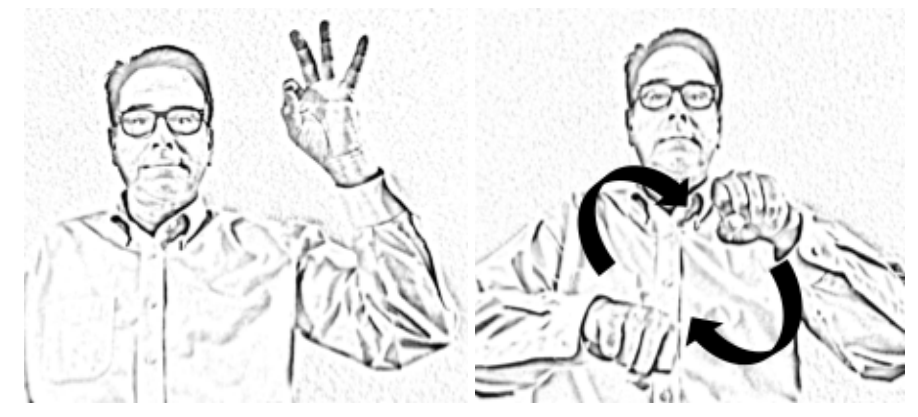

idence that supports the important status of contacted locations and by extension the ensuing canonical posture. We discuss the special linguistics status of contacted locations, describe how these factor in the execution and articulation of signs (see also Wilbur 2010). We then move to consider the role of postures in sign language recognition.

\subsection{Internal grammatical evidence}

Using the ASL-LEX 2.0 (Sehyr et al. 2021), we can begin to make some general claims about the prevalence of signs with canonical postures. ASL-LEX 2.0 lists $\approx 2,700$ ASL signs, limiting entries to simplex ASL forms (i.e., excluding morphologically compound signs, fingerspelled and initialized signs), 2151 entries are listed, with 1311 that have contact and 840 without contact, representing approximately 3:2 ratio. It is of additional interest to note that statistically the contacting signs are shorter in duration than non-contacting signs ( $p$ $>.001$ ). At first blush this indicates that postural forms are productive and perceptually efficient. There are semantic differences as well where non-contacting signs are statistically more likely to be rated as highly iconic relative to the contacted signs with postural forms. Forging an understanding of signs that meet the definition of postural forms will go quite far in accounting for a major formational property of the ASL lexicon.

Not only do signs that exhibit a canonical posture constitute a majority of sign forms in the ASL lexicon, there is evidence that the grammar makes special reference to instances of contacted locations within signs. Morphological processes such a compound formation reveals this special status. Compounds are understood as being derived from two separate lexical forms which are lawfully restructured into a unified compound. An example includes forms like SISTER which is derived from the ASL signs for GIRL and SAME. The resultant 
compound that surfaces exhibit considerable restructuring of the input forms. There have been several formal accounts (see for example Liddell and Johnson 1986; Sandler 1989). Regardless of particular theoretical treatment, there is agreement that during compound formation certain movements and components of the input signs are lost while others are faithfully retained. A generalization that has emerged from this work is that it is typically the contacted location of the input sign form(s) that is/are retained in ASL compounds. For example, in the sign SISTER, the input sign GIRL loses repetition as does the canonical form of the sign SAME. Importantly the resulting compound retains an initial posture in which the thumb makes contact with the cheek and a final posture where the index fingers of the dominant and non-dominant hand contact (note in modern forms there is additional coarticulatory restructuring of orientation and handshape, but this is not relevant to the present discussion). The central point is that the grammar must afford a special status to canonical postural forms that remain in the restructured well-formed compound.

\subsection{Sign language production}

Not only is the grammar of the language sensitive to postures, there is evidence from sign language acquisition and language breakdown that postural forms constitute foundational components of sign formation: they are robustly acquired and most resistant to disruption.

In a longitudinal study of three deaf children of deaf parents (ages 7 to 17 months), Conlin et al. (2000) provide a quantification of naturally occurring immature sign forms. These sign forms represent the child's deviation from the expected adult form of the sign (i.e., "babysigns"). In their corpus Conlin et al. report that overwhelmingly children learning sign language mis-produce expected handshapes (279/372 tokens) and movements (171/372 tokens) while place of articulation errors are far less common (69/372) and exhibit less variability. However, what is not reported is whether within the small number of POA errors made, is whether these errors involve canonical postures. There are some hints. First the PoA errors are more likely to occur within neutral space than involve location of the head or face. Moreover, many of these errors appear to be non-structure preserving, that is they are not substitution of phonologically contrastive POA for another, but in many instances are signs produced outside of articulatory sign space. I surmise that these are errors that are not anchored to a contacted body location. Moreover, they report instances where location "errors" arise in cases whether the child may have inadvertently rotated their head while in the middle of the execution of a sign. These types of performative "errors" suggest that the child's internal model correctly encoded the intended target location of the sign, but there was difficulty coordinating the linguistic and non-linguistics movements (Conlin et al. 2000).

There are two important generalizations. First, as reported by Conlin et al. (2000), the ability to produce the correct place of sign articulation (i.e., location) in infants learning ASL is much better preserved than the attainment of correct handshape or movements. Moreover, when POA errors do occur, they frequently (though not exclusively), are signs that are not articulated on the body. These data suggest that sign forms that lack a canonical posture may be more error-prone, than signs that exhibit a canonical posture. Here I postulate that a contacted location provides a potent somatic cue during sign production, one that enhances the correct achievement of a sign (see also Marentette 1995). The importance of a contacted articulatory location is further evidenced in cases where a parent may occasionally sign on a child's own body. As noted by Holzrichter (1995) a parent whom she observed produced the sign DADDY in contact with her daughter's forehead. The place of articulation, albeit transferred to the child, was correct, but the handshape that the mother produced was 
an index-hand rather than the expected 5-hand. Native signing parents appear aware that achieving contacted locations holds special merit in a child's acquisition of the language.

The prominence of a contacted location is also observed in paraphasia errors of deaf signers with stroke. As detailed in Corina (2013), handshape errors are very common in sign paraphasia while errors that displace the location of the sign are rare. An example of this can also be seen in a case of cortical stimulation in neurosurgical patient (Corina et al. 1999). For example, signer S.T. when asked to produce the ASL signs for COW and SHIRT, under stimulation of the left cortical opercular region the intended handshapes were commonly reduced to laxed closed fist forms and the precise path movements (e.g., wrist-twist for COW, a repeated tap for SHIRT) was replaced with relaxed tapping or rubbing movements. Importantly the signer was able to approximate the correct contacted location of the signs, the right temple and ipsilateral upper chest, normally contacted location in the signs COW and SHIRT respectively. Stimulation of the parietal opercular area also resulted in signs whose articulatory locations were preserved but errors in handshape and movement were noted. Of the 14 errors illustrated in Corina et al. (1999) that involve a contacted location, 13 examples preserved the contacted location.

Various researchers have commented on the relative integrity of signed locations observed in production studies of signed languages. In the acquisition data some explanations suggest that gross motor constraints on whole arm movements, which rely upon more proximal body joint (e.g., shoulder elbow) are easier to control relative to the distal fine-motor articulation of hands (Meier et al. 1998). However, this motoric explanation cannot be the sole determinant of preserved contact. Note that while some aphasics evidence a hemiplegia affecting the upper limb contralateral to the damaged hemisphere, sign language paraphasia's (with preserved contact) still occur on the non-motorically involved limb. Other explanations offered suggest that articulatory locations may be more perceptually salient than say particular configuration of handshapes and hence are learned and reproduced earlier. In the case of sign disruptions observed in aphasic productions or induced by direct brain stimulation, under these sub-optimal communicative conditions, achieving an approximated postural form is most efficacious in an otherwise compromised communicative exchange.

A separate deaf signing neurosurgical patient provides further evidence of the importance of location information during signing and clues to neural substrates of postures. In a recent study electrocortical activity was recorded while an awake participant produced and copied signs and pseudo-signs (Leonard et al. 2020). High-gamma brain activity (HG; 70$150 \mathrm{~Hz}$ ) recorded across at electrodes in left hemisphere pre- and post-central gyrus and the supramarginal gyrus (SMG) was significantly correlated with the execution of location and handshape features. With respect to location, somatotopically organized cortical regions differentiated contacted locations to the face and the non-dominant hand from non-contacted articulatory neutral space and fingerspelling space. Notably some electrodes showed sharp responses that were locked to the onset of movement (the video frame when the participant begins to move his hand for the corresponding movement/feature) while others showed more sustained responses that began prior to movement onset. The latter response type is typical of motor evoked activity, which reflects preparation and planning that precedes the actual movement. Classification of neural activity related to location features was broad and peaked prior to movement onset, in contrast to handshape features which peaked following movement onset. What's more, when we considered separately signs that exhibited physical location contact, from instances where the patient produced a sign that is specified for contact, but in performance at the time did not happen to achieve physical body contact, the neural responses were the same. This evidence suggests an abstract property of location, 
and I would argue posture, plays an important role in the planning of sign movements, a central tenet of postural account of signing.

In summary, we have seen that canonical posture serve as a potent cue in sign formation. Signs that contain canonical postures are well represented in the ASL lexicon, the special status of contacted locations is recognized by the grammar of the language, immature articulatory sign forms produced by deaf signing infants and articulatory forms evidenced in paraphasic signing tend to preserve contacted locations. Neural recording during sign articulation show evidence that contacted location may serve as an important planning unit in movement executing during signing. These facts suggest that canonical postures maybe a central organizational principle in sign formation and in sign production. Next, we consider the role of canonical postures in sign language recognition.

\section{Postures in sign recognition}

\subsection{Sign recognition is efficient.}

Recognition of the signs rely upon the accumulation of visual sensory information that unfolds in time. While measurements vary, it is commonly accepted that the speed taken to utter a mono-syllabic spoken word is either shorter or at best no longer than the time required to produce a monosyllabic sign. The common explanation for these differences is rooted in the physical differences in articulators where the ballistic movements of the limbs are larger and require greater displacement than the oral articulators. Despite the durational differences a striking finding is that relative to speech, signers need far less of the total sign-signal to identify signs. Several studies have used gating procedures to quantify these differences in recognition and identify the time course of recognition for signing (Grosjean 1981; Emmorey and Corina 1990; Morford and Carlson 2011). In these studies signers are shown snippets of signs as they unfold in time. Subjects are encouraged to guess the identity of a sign at each "gate". One can then assess the time it takes to identify the target signal relative to duration of the sign. Studies by Emmorey and Corina (1990) report that sign isolation required approximately $35 \%$ of the sign before the sign was identified. In comparison spoken word recognition in English required $83 \%$ of the spoken word to be heard before the subject could identify the comparable spoken word. How this rapid assessment of the sign signal occurs is not fully understood, however there are clues.

A consistent finding across these studies is that signers first seize upon information about the place of articulation (i.e., location) and when tested, the orientation of the sign prior to identifying handshape and movements ${ }^{3}$ (Clark and Grosjean 1982; Emmorey and Corina 1990; Grosjean 1981; Morford and Carlson 2011). However, the notion of place of articulation (i.e., location) used in these studies is fairly broad, as location includes signs articulated in neural space that lack bodily contact. Crucial to my argument however is that postural forms, that include physical contact between the hand(s) and body may influence the rapidity of sign recognition. A re-examination of the data presented in Grosjean (1981) reveals the following pattern, while reported durations of the sign with contact or no contact are not statistically different, the times to isolate the signs with postural contact are significantly

3. Morford and Carlson (2011) reported both location and handshape were identified equally fast in their gating study. However, as they note the first gate consisted of four frames, or $128 \mathrm{~ms}$, whereas prior studies used a first gate consisting of one frame, or $33 \mathrm{~ms}$. This methodological change may have biased the assessment of early stages of sign recognition. 
shorter than the isolation times for signs without contact. (See Tables 2 and 3 below $^{4}$ ). As duly noted in the original Grosjean (1981) article, while a host of factors may conspire to influence these isolation times (sign frequency, frequency of sign location, number of repetitions, distance from the starting position of the hands to the sign location, sign complexity, movement complexity, sign duration) much of the variance of isolation times still remains to be accounted for. My reassessment of the original data suggests that postural contact may provide a further accounting of the variance. What is important to note is that sign isolation is shorter than the measured duration of the sign. This strongly suggests that signers are making predictions about of the appropriate location (POA) while they are perceiving signs in real time.

Table 2: Global assessment of all sign types without and with contact

\begin{tabular}{llll} 
& Signs & Duration & Isolation \\
\hline Without contact & $\mathrm{n}=10$ & 789.8 & 455 \\
With contact & $\mathrm{n}=24$ & 844.3 & 377 \\
T-tests (2-tailed) & & $\mathrm{p}<.43$ & $\mathrm{p}<0.45$ \\
\hline
\end{tabular}

Table 3: Conservative assessment of one-handed signs without and with contact

\begin{tabular}{llll} 
& Signs & Duration & Isolation \\
\hline Without contact & $\mathrm{n}=5$ & 850.2 & 515 \\
With contact & $\mathrm{n}=13$ & 782.1 & 383 \\
T-tests (2-tailed) & & $\mathrm{p}<.47$ & $\mathrm{p}<0.1$ \\
\hline
\end{tabular}

\subsection{Sign recognition is holistic.}

A postural account of sign recognition proposes that aspects of sign recognition make use of configural processing, an assessment of the global properties of a sign, rather than a commonly accepted analyses-by-synthesis account whereby sign recognition requires a deconstructed componential feature-based assessment of handshape, orientation, movement and place of articulation. The evidence for a reliance on configural over featural processing is based, in part, on stimulus inversion effects, whereby the $180^{\circ}$ inversion of particular class of stimuli adversely affects the processing of those stimuli. Inversion disrupts the appreciation of the canonical relationships between features of the stimuli (Maurer, Grand, and Mondloch 2002). In addition to stimulus characteristics, perceiver characteristics such as expertise are known to modulate inversion effect (Gauthier and Tarr 1997; Gauthier et al. 1998).

Studies of the effect of inversion on the recognition of signing in native signers and sign naïve individuals provides evidence for the role of configural processing and the expertise in sign language recognition. Corina and Grosvald (2012) used a continuous categorization

4. In the absence of images or videos of the signs used, I made the following decisions. In the global measures sign with two-handed signed in neutral space were treated as contacted/postures (e.g. COFFEE, MAKE, TRAIN). In the conservative analysis, only one-handed signs that in adult pronunciation are signed without a physical contact to body were included (e.g. PEPPER, SEARCH, THINGS). Signs that might be considered ambiguous as to whether they have physical contact (e.g. SUMMER, BLACK) were excluded. Note the counts for the distribution of contacted versus non-contacted signs in these analysis approaches the 3:2 contact to no-contact ratio reported noted in the corpus ASL-Lex corpus study. 
paradigm to explore these effects. In this paradigm subjects saw videos of ASL signs or selfgrooming (SG) gestures (e.g., scratching one's head, rubbing an eye etc.) and decided as quickly as possible if the video depicted an ASL sign or a non-linguistic action. To make a proper classification, a subject must minimally identify the distinguishing properties of these two classes of stimuli across many exemplars. Subjects saw both upright and inverted instances of ASL signs and SG gestures. Examining the effect of inversion on the sign trials, we observe that the categorization of inverted signs was relatively more taxing for the deaf signers compared to sign naïve participants. Planned comparisons indicated that the inversion effects for signs were statistically more detrimental for the deaf signers compared to hearing subjects (deaf signers $=65 \mathrm{msec}$, hearing sign-naive participants $=32 \mathrm{msec} \mathrm{p}<$ 0.001). This degree of disruption is particularly notable in that deaf signers' overall categorization judgements were roughly $100 \mathrm{msec}$ faster than the hearing subject's judgements (mean RT = $758 \mathrm{~ms}$ for deaf and $868 \mathrm{~ms}$ for hearing). As discussed in Corina and Grosvald (2012), this overall difference suggests that deaf signers show a perceptual expertise for recognizing human actions (both linguistics and non-linguistic). We argue that taxing effects of inversion on sign perception in deaf signers reflects the relatively more configural appreciation of ASL by experts.

ASL lexical decision experiments also speak to the importance of locations during sign language recognition. Experiments have varied the formational similarity between sign pairs to explore the role of phonological components of sign recognition. A recurring finding in this literature is that the ability to accept a sign as a legal ASL sign is inhibited, if the preceding prime shares an articulatory location with the target. The effects of shared location were highlighted in an event related potential study reported by Gutierrez et al. (2012). In this experiment subjects were shown semantically constraining ASL sentences that could end in one of five possible ways. In the baseline condition, a logical semantic sign is observed (e.g., DRESS). In an unrelated condition, a semantically incongruous sign that shares no articulatory features of the intended target is seen (e.g., MIRROR). In a phonological condition, a semantically incongruous sign is seen that happens to share an articulatory location with the expected sign (e.g., LUNGS). Further continuations included a semantically congruent form that does not share an articulatory location of the intended target (e.g., SKIRT). And finally, a semantically congruous form that shares an articulatory location with the expected sign (e.g., SHIRT). An example of a sentence and its continuations is shown below.

ME FRIEND WEDDING HERS ME BRIDESMAID ME WEAR MUST EXCITED ME

a. Semantically congruent DRESS

b. Semantically incongruent no shared location MIRROR

c. Semantically incongruous shared location LUNGS

d. Semantically congruent no shared location SKIRT

e. Semantically congruent shared location SHIRT

As was expected from prior studies, relative to the baseline, we observe an electrophysiological N-400 effect -a significant negative deflection between 300-600 milliseconds after presentation of the target sign, for the semantically incongruent sign MIRROR. This N400 effect indexes the difficulty in integrating the target into the developing semantic representation conveyed by the sentence. However, what is particularly striking is when the semantically incongruent sign (e.g., LUNGS) which shares a phonological location [chest] with the semantically congruent target (i.e., DRESS), we observe an earlier robust negative deflection. We argue that this reflects the added difficulty to abandon a lexical competitor when it shares a common articulatory location with the expected sign. Just as with data from lexical decision 
experiments, these data reinforce the notion that shared location or place of articulation is a salient property in organization of lexicon, and organizational principle that has processing consequences.

\subsection{Perceptual sensitivity to postures}

As mentioned in the discussion of the gating data, it is interesting to note that in addition to the parameter for location being first identified, that hand orientation is also correctly appraised early-on the sign identification process. This provides a hint that the perception of body postures may include relational information about limb orientation. In a recent neuroimaging study (Almeida, Poeppel, and Corina 2016) we have reported that Deaf signers are particularly adept at noticing violations of body postures. In this experiment we used MEG technique to document neuromagnetic signal associated with early processing of signs. We presented deaf signers and hearing non-signers, selected still images of sign forms. These still images largely represented canonical sign postures and were easily identified as lexical signs in the absence of path movements. To create body-form violations, we carefully altered these images such that the left and right hands of the signers were swapped (see Figure 3 ). On first glance, these images appear to represent familiar signs, however a more careful inspection reveals that these manipulated images are biologically impossible. Both deaf signers and hearing naïve signers can make this assessment. What was particularly striking was the deaf signers' discrimination of these forms as assessed by D' measures, were significantly higher than hearing non-signers. Even when controlling for reaction time, Deaf signers showed significantly better discrimination of these forms relative to sign-naive participants. The MEG responses further identified significant neuromagnetic signatures which differentiated deaf and hearing subjects. Specifically, an early M130 component attributed to occipital temporal sources reflected an early specialization in the deaf signers that drives these form-based judgments.

Figure 3: Biologically plausible (left) and implausible (right) sign postures.
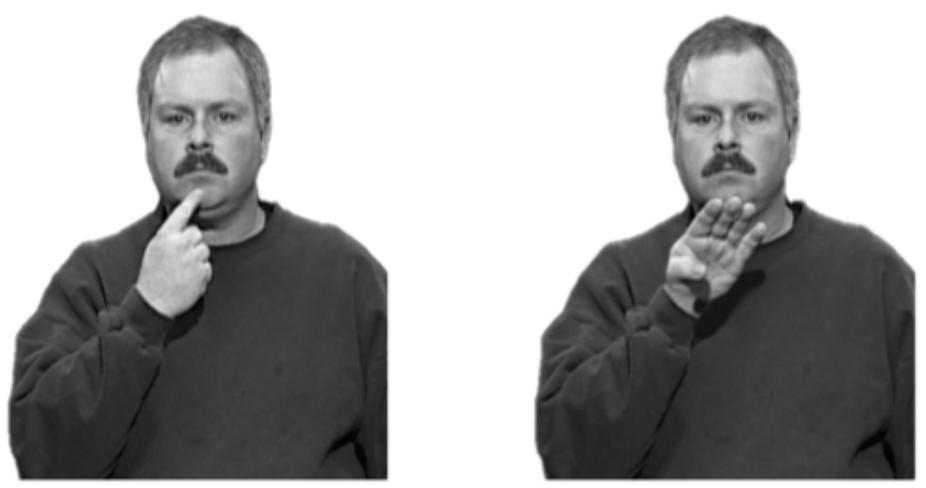

\section{Sign language postures and forward models}

A long-standing issue in the domain of motor control asks how humans faithfully maneuver in a complex environment and how bodily movements are organized to interact with objects in our environment. Research in this area has proposed that accuracy and adaptability of body movements are achieved through the use of a forward model. Forward models use an efference copy of the motor command to make a prediction of the consequences of the motor act. A forward output model makes predictions about the sensory consequences of 
the movement and this prediction is compared with the actual sensory consequences of a movement (Miall and Wolpert 1996). Current theories of speech motor control emphasize the importance of state-feedback control (Hickok, Houde, and Rong 2011; Houde and Nagarajan 2011), including forward models of action selection and error correction (Wolpert and Flanagan 2001). In the domain of language, forward models have recently been invoked as a mechanism for language comprehension. Pickering and Garrod (2007) suggest that the production system acts as an emulator during language comprehension. They argue that a comprehender uses prediction and imitation to construct an 'emulator' using the production system, and combine predictions with the input dynamically. In their model such emulators may serve different levels of language form, from phonology to semantics.

The accumulating evidence presented here is consistent with the idea that postural forms may provide an important component in the organization of skilled linguistic movements that we see in signed languages. The evidence strongly suggests that contacted location, or postures more generally are crucial and may serve as articulatory targets in sign production. In the domain of sign recognition, signers actively predict sign location (and orientation) in studies of sign gating and show evidence of competition among signs that share postural states. Moreover, psycholinguistic and neuromagnetic evidence indicates that skilled signers may be taking a more wholistic parse of the sign signal, one that is quietly sensitive to violation of possible biological postures. These facts I argue are consistent with the premise that psychological and neural underlining of sign language understanding may make use of forward models and that postural forms play a central role in the on-going prediction of possible signs and may further guide the skilled articulation of these forms.

Figure 4: A forward model of sign language processing incorporating abstract articulatory postural forms in the evaluation of signing.

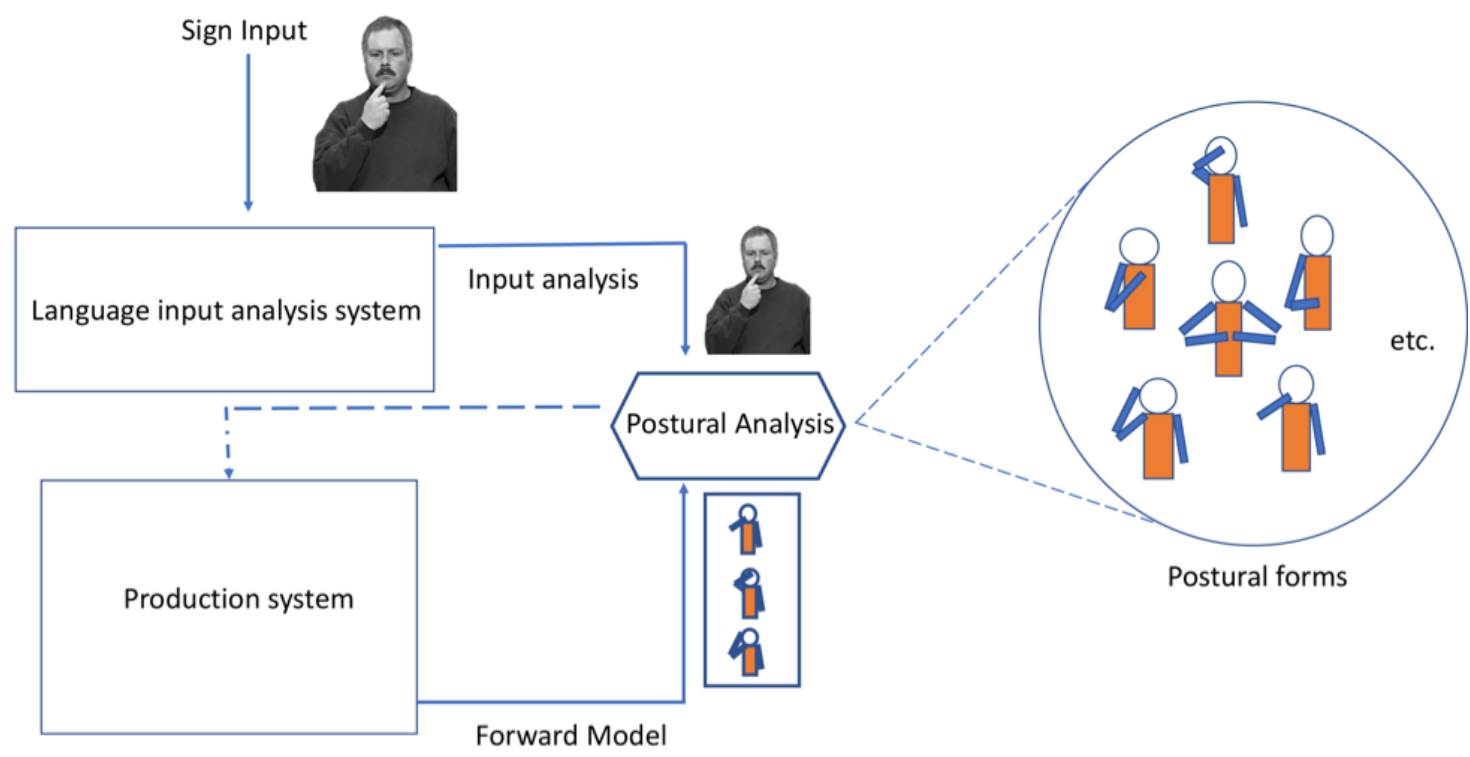

In signed languages postural forms serve as a planning/evaluative unit for sign language understanding

In Figure 4 I illustrate a schematic diagram what indicated how postural form may inform forward model of sign processing. It is important to note that this is but one aspect of a more complete model whereby additional information including additional articulatory, morphological and syntactic, information may lead to the rapid and exquisite deployment 
and recognition of skilled signing. In production, postural sign forms serve as global targets for sign articulation and guide the execution of the form. In comprehension, I contend that similar postural targets are used in the service of sign recognition whereby the accruing sensory is compared against a predicted forward internal model to guide the efficient recognition of signs and or detect violations. This account differs from popular analysis-bysynthesis componential models of sign recognition which dominate current psycholinguistic literature.

\section{References}

Almeida, Diogo, David Poeppel, and David Corina. 2016. "The processing of biologically plausible and implausible forms in American Sign Language: evidence for perceptual tuning." Language, Cognition and Neuroscience 31 (3): 361-374. https: / / doi . org / 10. 1080/23273798.2015.1100315.

Battison, Robbin. 1978. Lexical borrowing in American Sign Language. Silver Spring: Linstok Press.

Channon, Rachel. 2002. "Beads on a string? Representations of repetition in spoken and signed languages." In Modality and structure in signed and spoken languages, edited by Richard P. Meier, Kearsey Cormier, and David Quinto-Pozos. Cambridge: Cambridge University Press.

Clark, Lorene E., and François Grosjean. 1982. "Sign recognition processes in American Sign Language: the effect of context." Language and Speech 25 (4): 325-340. https:// doi.org/ $10.1177 / 002383098202500402$.

Conlin, Kimberly E., Gene R. Mirus, Claude Mauk, and Richard P. Meier. 2000. "The acquisition of first signs: Place, handshape, and movement." In Language acquisition by eye, edited by Charlene Chamberlain, Jill P. Morford, and Rachel I. Mayberry, 51-69. Lawrence Erlbaum Associates.

Corina, David. 2013. "Some observations regarding paraphasia in American Sign Language." In The Signs of Language Revisited: An Anthology To Honor Ursula Bellugi and Edward Klima, edited by Karen Emmorey and Harlan L. Lane, 414-426. Psychology Press. https: //doi.org/10.4324/9781410604972.

Corina, David P., and Michael Grosvald. 2012. "Exploring perceptual processing of ASL and human actions: Effects of inversion and repetition priming." Cognition 122 (3): 330-345. https://doi.org/10.1016/j.cognition.2011.10.011.

Corina, David P., Susan L. McBurney, Carl Dodrill, Kevin Hinshaw, Jim Brinkley, and George Ojemann. 1999. "Functional roles of Broca's area and SMG: Evidence from cortical stimulation mapping in a deaf signer." NeuroImage 10 (5): 570-581. https://doi.org/10.1006/ nimg.1999.0499.

Emmorey, Karen, and David Corina. 1990. "Lexical recognition in sign language: Effects of phonetic structure and morphology.” Perceptual and Motor Skills 71 (3): 1227-1252. htt ps://doi.org/10.2466/pms.1990.71.3f.1227. 
Gauthier, Isabel, and Michael J. Tarr. 1997. "Becoming a "greeble" expert: Exploring mechanisms for face recognition." Vision Research 37 (12): 1673-1682. https: / / doi.org / 10. 1016/S0042-6989(96)00286-6.

Gauthier, Isabel, Pepper Williams, Michael J. Tarr, and James Tanaka. 1998. "Training 'greeble' experts: a framework for studying expert object recognition processes.” Vision Research 38 (15): 2401-2428. https://doi.org/10.1016/S0042-6989(97)00442-2.

Grosjean, François. 1981. “Sign \& word recognition: A first comparison.” Sign Language Studies 32:195-220.

Gutierrez, Eva, Deborah Williams, Michael Grosvald, and David Corina. 2012. "Lexical access in American Sign Language: An ERP investigation of effects of semantics and phonology." Brain Research 1468:63-83. https://doi.org/10.1016/j.brainres.2012.04.029.

Hickok, Gregory, John Houde, and Feng Rong. 2011. "Sensorimotor integration in speech processing: Computational sasis and neural organization.” Neuron 69 (3): 407-422. http s://doi.org/10.1016/j.neuron.2011.01.019.

Holzrichter, Amanda S. 1995. “Motherese in American Sign Language.” MA thesis, University of Texas in Austin.

Houde, John F., and Srikantan S. Nagarajan. 2011. "Speech production as state feedback control.” Frontiers in Human Neuroscience 5. https:// doi.org/10.3389/fnhum.2011.00082.

Leonard, Matthew K., Ben Lucas, Shane Blau, David P. Corina, and Edward F. Chang. 2020. "Cortical encoding of manual articulatory and linguistic features in American Sign Language.” Current Biology 30 (22): 4342-4351.e3. https: / / doi.org/ 10.1016/j.cub.2020.08. 048.

Liddell, Scott K., and Robert E. Johnson. 1986. "American Sign Language compound formation processes, lexicalization, and phonological remnants." Natural Language and Linguistic Theory 4 (4): 445-513. https://doi.org/10.1007/BF00134470.

Marentette, Paula F. 1995. "It's in her hand: A case study of the emergence of phonology in American Sign Language.” Doctoral dissertation, McGill University.

Maurer, Daphne, Richard Le Grand, and Catherine J. Mondloch. 2002. "The many faces of configural processing." Trends in Cognitive Sciences 6 (6): 255-260. https: / / doi.org/ 10. 1016/S1364-6613(02)01903-4.

Meier, Richard P., Claude Mauk, Gene R. Mirus, and Kimberly E. Conlin. 1998. "Motoric constraints on early sign acquisition." In Papers and reports on Child Language Development, edited by Eve V. Clark, 29:63-72. Stanford: CSU Publications.

Miall, Chris R., and Daniel M. Wolpert. 1996. "Forward models for physiological motor control.” Neural Networks 9 (8): 1265-1279. https:// doi.org/10.1016/S0893-6080(96)000354.

Morford, Jill P., and Martina L. Carlson. 2011. "Sign perception and recognition in non-native signers of ASL.” Language Learning and Development 7 (2): 149-168. https:// doi.org/10. 1080/15475441.2011.543393. 
Pickering, Martin J., and Simon Garrod. 2007. "Do people use language production to make predictions during comprehension?” Trends in Cognitive Sciences 11 (3): 105-110. https: //doi.org/10.1016/j.tics.2006.12.002.

Sandler, Wendy. 1989. Phonological representations of the sign. Dordrecht: Floris.

Sandler, Wendy, and Diane C. Lillo-Martin. 2006. Sign language and linguistic universals. Cambridge: Cambridge University Press.

Sehyr, Zed Sevcikova, Naomi Caselli, Ariel M Cohen-Goldberg, and Karen Emmorey. 2021. "The ASL-LEX 2.0 project: A database of lexical and phonological properties for 2,723 signs in American Sign Language.” The Journal of Deaf Studies and Deaf Education 26 (2): 263-277. https://doi.org/10.1093/deafed/enaa038.

Stokoe, William. 1960. Sign language structure. Studies in linguistics: Occasional papers 8. Buffalo: Department of Anthropology and Linguistics, University of Buffalo.

Wilbur, Ronnie B. 2010. “The role of contact in the phonology of ASL." Sign Language \& Linguistics 13 (2): 203-216. https://doi.org/10.1075/sll.13.2.05wil.

Wolpert, Daniel M., and Randell J. Flanagan. 2001. "Motor prediction.” Current Biology 11:729732. 